\title{
Solving the conundrum of cholangiocyte differentiation
}

Two new papers published in Nature

Biotechnology have described methods for generating cholangiocyte organoids from human pluripotent stem cells.

Biliary diseases account for a substantial proportion of liver transplantations in adults and children, but the progress in developing new therapies has been hampered by a lack of research tools, namely, cell lines and animal models.

Both groups differentiated cholangiocytes from stem cells: Ogawa et al. from human embryonic and induced pluripotent stem cells and Sampaziotis et al. from human induced pluripotent stem cells. The cholangiocyte organoids were subsequently assessed for their use as systems for disease modelling and drug screening.

Stem cells were induced to differentiate into hepatoblasts and then cholangiocytes in different ways. Sampaziotis et al. used activin, retinoic acid and fibroblast growth factor-10, whereas Ogawa et al. activated the NOTCH pathway and added growth factors in culture. These methods led to a high percentage of cells expressing cholangiocyte markers (such as cytokeratin 7 and 19). Both groups used 3D culture methods to further mature the cholangiocyte-like cells, leading to the formation of organoids with tubular and cystic morphology (see image).

Luminal accumulation of rhodamine 123, a fluoroescent substrate for the cholangiocyte transporter multidrug resistance 1 (MDR1), showed that the organoids were functional.

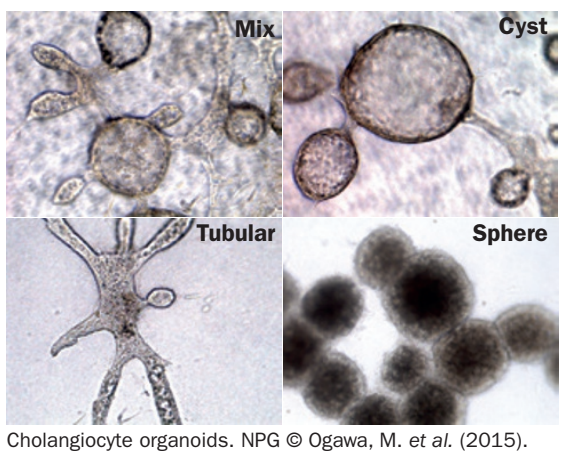

Ogawa et al. implanted the organoids subcutaneously within a Matrigel plug in immunodeficient mice and 6-8 weeks later ductal structures were found, which expressed cytokeratin 19 and other biliary markers. Sampaziotis et al. analysed transcriptomic data from their cholangiocyte-like cells, which clustered closely with primary cholangiocyte data.

Finally, cholangiocyte organoids were sucessfully cultured from patients with cystic fibrosis, a condition that affects secretory epithelial cells such as cholangiocytes, causing liver disease. The authors hope that these organoids can be used to further understand disease mechanisms and develop new drugs.

Gillian Patman

Original articles Sampaziotis, Fe et al. Cholangiocytes derived from human induced pluripotent stem cells for disease modeling and drug validation. Nat. Biotechnol. doi:10.1038/nbt.3275 | Ogawa, M. et al. Directed differentiation of cholangiocytes from human pluripotent stem cells. Nat. Biotechnol. doi:10.1038/nbt.3294 